\title{
Damage characterizations and simulation of selective laser melting fabricated 3D re-entrant lattices based on in-situ CT testing and geometric reconstruction
}

\author{
Luchao Geng a , Wenwang Wu ${ }^{\text {b,c,*, Lijuan Sun }}{ }^{\mathrm{d}}$, Daining Fang ${ }^{\mathrm{a}, \mathrm{b}, *}$ \\ a State Key Laboratory for Turbulence and Complex System, Department of Mechanics and Engineering Science, College of Engineering, Peking University, Beijing \\ 100871, China \\ ${ }^{\mathrm{b}}$ Institute of Advanced Structure Technology, Beijing Institute of Technology, Beijing 100081, China \\ ${ }^{\mathrm{c}}$ Department of Mechanical Engineering, Massachusetts Institute of Technology, Cambridge, MA 02139, USA \\ d The State Key Laboratory of Nonlinear Mechanics, Institute of mechanics, Chinese academy of sciences, Beijing, China, 100190
}

\section{A R T I C L E I N F O}

\section{Keywords:}

Selective laser melting

Computed tomography

Image finite element

Re-entrant

In-situ mechanical test

\begin{abstract}
A B S T R A C T
In recent years, metal additive manufacturing (AM) are widely employed for industrial applications, such as: biomedical, aerospace, automotive, marine and offshore sections. AM demonstrated superior manufacturing efficiencies and economic advantages for advanced lightweight industrial components with unlimited arbitrary topological layouts and complex internal microstructures, and are also employed for fabrication of auxetic materials and structures. In this paper, damage characterizations and mechanical behaviors of selective laser melting (SLM) fabricated 3D re-entrant lattices are investigated based on in-situ interrupted micro-CT test, and simulation based on geometric reconstructed models are performed for exploring the underlying failure mechanisms. Firstly, theoretical models for predicting the mechanical properties of 3D re-entrant lattice are developed, such as stiffness, Poisson's ratio and strength, etc. Secondly, the geometrical errors and fabrication defects of 3D reentrant lattices are analyzed based on 3D micro-CT scanning, in-situ micro-CT interrupted compression tests are performed for studying the deformation process and failure mechanisms. Finally, image finite element models with the detailed information of the shape, position and distribution of defects of the 3D reentrant lattices are constructed from 3D tomographic images, and numerical simulations are performed for studying the effects of the defects on the mechanical performances of the SLM additive manufactured 3D re-entrant lattice structures. It is shown that the failure behavior of the reentrant lattice is governed not only by its topology, but also by the geometric defects and surface defects. Moreover, the proposed interrupted in-situ micro-CT mechanical loading experiments and image finite element approaches can also shed lights on the relations between fracture failure around the edge and the powder adhesion. The damage evolution process is compared with the numerical simulation results to verify the materials failure modes.
\end{abstract}

\section{Introduction}

Auxetics are structures or materials that have a negative Poisson's ratio (NPR), and are also known as materials with anti-rubber or dilational features. Different from traditional materials, auxetics will be elongated along directions perpendicular to the tensile loading direction. Auxetic materials with NPRs have the following special properties: high shear modulus, high energy absorption rate, and heat insulation, etc. [1-3]. Such excellent physical properties play an important role in realizing multi-functional performances, and are of great significance for the development of aerospace, semiconductor devices, optical components, precision instruments, biomaterials and building materials [4-7].
In recent years, metal additive manufacturing (AM) are widely employed for industrial applications, such as: biomedical, aerospace, automotive, marine and offshore sections. AM demonstrated superior manufacturing efficiencies and economic advantages for advanced lightweight industrial components with unlimited arbitrary topological layouts and complex internal microstructures. Negative Poisson's ratio structures are difficult to manufacture by traditional processes, and various types of advanced metal additive manufacturing techniques are proposed for fabrications of auxetic materials and structures due to their complex topology, such as selective laser melting (SLM) manufacturing of metal auxetics. Despite the huge progress in recent years in metal AM technologies, there remain some production issues which

\footnotetext{
* Corresponding authors at: Institute of Advanced Structure Technology, Beijing Institute of Technology, Beijing 100081, China.

E-mail addresses: wuww05@mit.edu (W.Wu), fangdn@pku.edu.cn (D. Fang).
} 
undermine the mechanical integrity of as-fabricated industrial components, such as stiffness, strength, ductility, corrosion, and fatigue resistances. In practice, metal AM inevitably introduces surface and internal defects into the manufacturing structures with complex configurations, the most common defects are unwanted porosity from incorrect processing parameters or build conditions, surface roughness or other surface imperfections, residual stresses and resultant deformation caused by the complex thermal history, and anisotropic mechanical properties, which are closely related to laser speed, laser energy, scanning path strategy and powder thickness, etc. Moreover, these manufacturing process induced imperfections can be exaggerated due to the complex nature of the designs, and industrial applications of additive manufactured industrial components are still limited due to the degradation of mechanical integrity under complex static and dynamic external mechanical loading environment.

The Selective Laser Melting (SLM) is an additive manufacturing technology that uses metal powders and 3D CAD files to produce a quasifinished product from a powder bed $[8,9]$. There are four major defects detected in the aluminum alloy components which are fabricated by SLM: porosities, surface quality, defects related to thermal cracking phenomena and anisotropy induced in the material [10]. Galy et al. [10] summarized the main defects of the aluminum alloy parts manufactured by SLM, and the causes for their forming and the consequences. Leon et al. [11] pointed out that surface roughness accelerates the fatigue life of specimens made by SLM. Tian et al. [12] found that it was helpful to reducing the surface roughness using the strategies of contour scan and skywriting scan. Furthermore, there is a more serious type of defect that is a geometric defect deviates from the design size. These defects have a significant impact on the mechanical properties and failure mechanisms of the lattice structure. In order to clarify these effects, $\mathrm{X}$-ray computed tomography (CT) as an important 3D non-destructive testing technology plays an important role in researches. In-situ CT has been used to study the influence of defects on the mechanical properties of metallic materials and failure mechanisms in metals by tracking defect distribution and damage evolution [13,14]. More and more researchers use CT technology to characterize the morphology of lattice structure made by additive manufacturing and reveal the relationship between mechanical properties and 3D quantification of structure quality [15-27]. Boniotti et al. [19] used digital image correlation to measure full-field strain of BCC lattice and compared with the finite element results obtained for the idealized and the real lattice geometries. Takano et al. [15] employed the first-order perturbation based stochastic homogenization method to categorize geometrical imperfections into kink, notch and hole and embedded them into finite element model to predict the reduction of stiffness. Carlton et al. [23] evaluated the failure mechanisms of two types of lattice structures by performing in-situ uniaxial compression tests to capture the local micro-strain deformation. Liu et al. [24] used CT to capture morphology, location, and distribution of process-induced defects and extracted the statistical distributions of three sets of defects from X-ray tomography images so as to study their role in the elastic response, damage initiation, and failure evolution under quasi-static compression. Amani et al. [25] built a 3D image-based conformal finite element model to simulate the compression test using Gurson-Tvergaard-Needleman (GTN) porous plasticity that is fairly good in agreement with experiments. There are also some representation methods of geometric imperfections recently. Wang et al. $[28,29]$ provided a benchmark of cylindrical shell for investigating the effect to knockdown factor from pure geometric imperfections which gave some guidance for studying the imperfection sensitivity of manufactured structure.

As concerns 3D re-entrant lattice structures, these materials manufacturing technologies [30-35] make it possible to fabricate. Yang et al. [36,37] established an analytical model of a 3D re-entrant lattice structure for getting analytical solutions of the elastic modulus, Poisson's ratios and compression yield strength of the re-entrant structure in all principal directions based on both a large deflection beam model and a Timoshenko beam model. Wang et al. [38] established an analytical model of a 3D re-entrant lattice structure based on energy method considering the overlapping of the struts as well as axial extension or compression. Li et al. [39] proposed a 3D augmented re-entrant cellular structure and derived its elastic properties in all principal directions. However, these theories do not give the critical compressive strength and the criteria for judging which strut failures firstly.

It can be seen from above literatures that no rigorous investigations have been carried out to further study the failure evolution of 3D reentrant lattice structure via in-situ CT and quantify the geometric errors between the real structure and the ideal model. In this study, theoretical models for predicting the mechanical properties of 3D re-entrant lattice are developed, such as stiffness, Poisson's ratio and strength, etc. Afterwards, we investigate the static compression response of 3D re-entrant lattice structures with ideal and real geometry and demonstrate the geometric defects induced by SLM, damage initiation, and failure evolution of the lattices using in-situ X-ray CT technique. The paper is organized in the following way. Analytical solution of the elastic properties and the critical compressive strength of 3D re-entrant lattice in principal direction are derived in Section 2. Section 3 demonstrates the manufacturing and microstructure of the lattice and presents the experimental results from in-situ X-ray computed tomography. Finally, numerical simulation elucidates the outcome from FEA of fully-detailed imperfect models in Section 4, which reveals the influence of defects on the mechanical response of 3D re-entrant lattices.

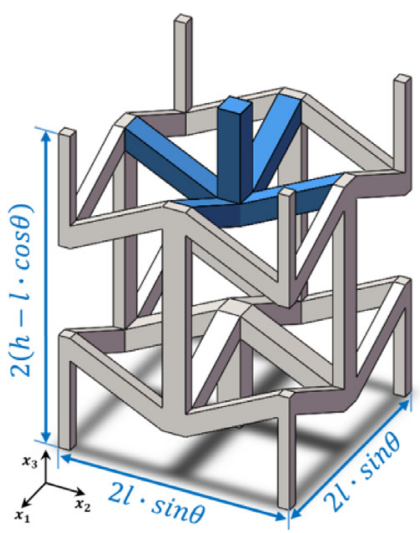

(a)

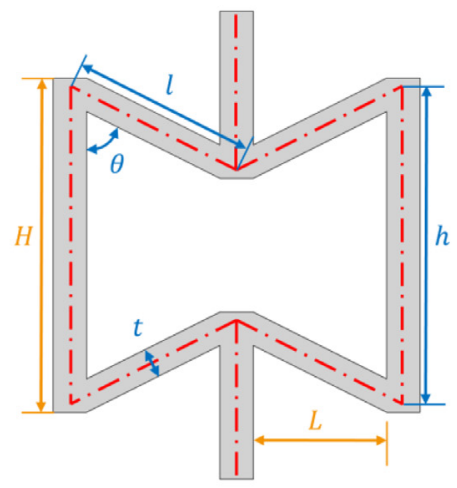

(b)

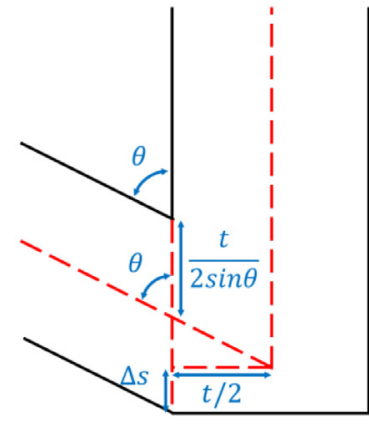

(c)
Fig. 1. (a) Unit cell of the 3D re-entrant structure, (b) geometry parameters, (c) geometric relations at the joint node. 

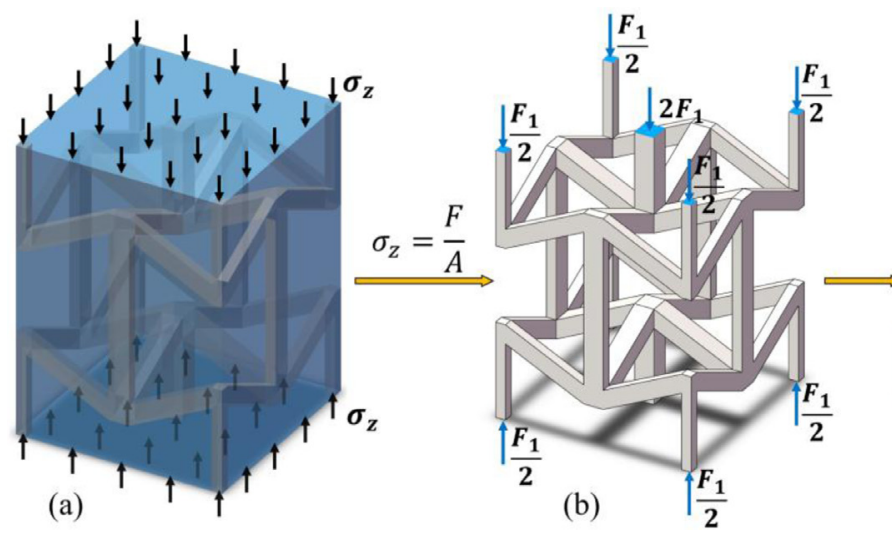

Fig. 2. (a) Stress on bulk material; (b) loading of cellular material; (c) loading of simplified 3D structure.

\section{Analytical model}

\subsection{Elastic properties}

The unit cell of the 3D re-entrant lattice structure is shown in Fig. 1(a). The cross section of all the struts both vertical and oblique are square. As shown in Fig. 1(b), there are four primary design parameters for the unit cell structure: the length of oblique strut $l$, the length of vertical strut $h$, the re-entrant angle $\theta$, and the side length of cross section of the strut $t$.

According to Gibson's theory [40], the ratio of the macroscopic density of the lattice structure to the density of the solid material is defined as the relative density:

$\rho_{r}=\frac{\rho_{c}}{\rho_{s}}=\frac{4 t^{2} H+16 t^{2} L}{(2 l \sin \theta)^{2} * 2(h-l \cos \theta)}$

In order to simplify the expression, the actual length $H$ of vertical strut and the internal distance $L$ between adjacent vertical strut were introduced, as shown in Fig. 1(b). Where $H$ and $L$ can be obtained by geometric relations shown in Fig. 1(c): $H=h+2 \Delta s=h+\frac{t(1-\cos \theta)}{\sin \theta}, L=$ $\left(l-\frac{t}{\sin \theta}\right) \sin \theta$.

Poisson's ratio and equivalent elastic modulus of re-entrant lattice under compressive stress in $x_{3}$ (referred as the ' $Z$ ') direction can be obtained from reference [36]:

$$
\begin{aligned}
& \nu_{z x}=-\frac{\cos \theta\left(\frac{h}{l}-\cos \theta\right)\left[\frac{(l-2 \Delta l)^{2}}{E_{s} t^{2}}+\frac{6}{5 G_{s}}-\frac{1}{E_{s}}\right]}{\frac{(l-2 \Delta l)^{2} \sin ^{2} \theta}{E_{s} t^{2}}+\frac{6 \sin \theta}{5 G_{s}}+\frac{\cos ^{2} \theta}{E_{s}}+\frac{4(h-2 \Delta h)}{E_{s}(l-2 \Delta l)}} \\
& E_{z}=\frac{\frac{t^{2}(h-l \cos \theta)}{l^{2} \sin ^{2} \theta}}{\frac{(l-2 \Delta l)^{3} \sin ^{2} \theta}{2 E_{s} t^{2}}+\frac{(l-2 \Delta l) \cos ^{2} \theta}{2 E_{s}}+\frac{3(l-2 \Delta l) \sin ^{2} \theta}{5 G_{s}}+\frac{2(h-2 \Delta h)}{2 E_{s}}}
\end{aligned}
$$

Where $E_{s}$ and $G_{s}$ are the elastic modulus and shear modulus of solid material, respectively. $\Delta l$ is the effective length reduction of the oblique strut, $\Delta h$ is the length reduction of vertical strut. Wang et al. [38] obtained the elastic properties of 3D re-entrant lattice, but did not derive the failure theory. Yang et al. [36] deduced the yield strength of the reentrant structure but did not consider the buckling of the vertical strut and the effects of struts overlapping. Based on Wang's theory, the critical compressive strength was deduced.

\subsection{Critical compressive strength}

Take lattice structure as bulk material, assuming that the compressive stress $\sigma_{z}$ is applied far from the infinite three-dimensional re-entrant lattice along $z$ direction(as shown in Fig. 2a), for a two-dimensional unit cell, the vertical strut is subjected to the compressive load $F_{1}$, and for a three-dimensional unit cell, the compressive load on the vertical strut is

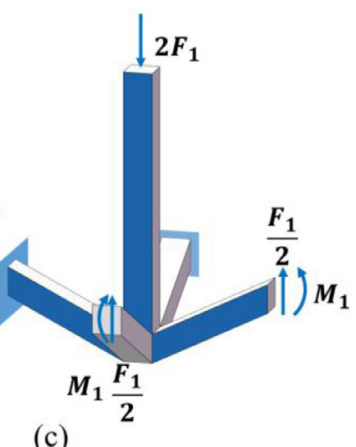

(c)

$2 F_{1}$ because the vertical strut is loaded on both $x-z$ and $y-z$ planes shown in Fig. 2c. So there are:

$\sigma_{z}=\frac{F}{A}=\frac{4 F_{1}}{(2 l \sin \theta)^{2}}=\frac{F_{1}}{l^{2} \sin ^{2} \theta}$

Under the compressive load, the vertical strut is prone to elastic buckling. According to the beam's Euler instability theory, assuming that both ends of vertical strut are fixed constraints, the critical compressive load for initial buckling is:

$$
F_{c r}=\frac{\pi^{2} E I}{[0.5(h-2 \Delta h)]^{2}}
$$

The load on the vertical strut in a three-dimensional cell is $2 F_{1}=F_{c r}$, For a square beam, the moment of inertia is $I=\frac{t^{4}}{12}$. Accordingly, in the case of macroscopic buckling of the vertical strut, ultimate strength $\sigma_{v b}$ applied far from the infinite 3D re-entrant lattice along $z$ direction can be expressed as:

$\sigma_{v b}=\frac{F_{c r}}{2 l^{2} \sin ^{2} \theta}=\frac{\pi^{2} E I}{6 l^{2} \sin ^{2} \theta(h-2 \Delta h)^{2}}$

The yield stress of solid material is $\sigma_{Y}$. When the compressive stress of vertical strut reaches the yield stress, the compressive load at this time is:

$2 F_{1}=\sigma_{Y} t^{2}$

Accordingly, in the case of macroscopic yield of the vertical strut, ultimate strength $\sigma_{v y}$ applied far from the infinite 3D re-entrant lattice along $z$ direction can be written in the following formulae:

$\sigma_{v y}=\frac{\sigma_{Y} t^{2}}{2 l^{2} \sin ^{2} \theta}$

For oblique strut, two assumptions were made: (1) Euler-Bernoulli's plane section assumption, that is, the plane perpendicular to the beam axis before deformation, remains plane after deformation, and perpendicular to the curved beam axis; (2) only the normal stress along the beam axis is important in the section, and the shear stress and the normal stress along the beam height direction causing the longitudinal extrusion are negligible.

Under the combined action of bending moment and axial force, the oblique strut reaches the plastic limit state. Under the plastic limit state, as shown in Fig. 3(d), the stress at any point in the beam is $+\sigma_{Y}$ or $-\sigma_{Y}$ except for the points of $\sigma=0$ on the neutral axis. Let the distance between the neutral axis and the geometric center axis of the cross section is $\eta t / 2$, then

$$
\begin{aligned}
& N=-\sigma_{Y} t \int_{-\frac{t}{2}}^{-\frac{\eta t}{2}} d y+\sigma_{Y} t \int_{-\frac{\eta t}{2}}^{\frac{t}{2}} d y=\sigma_{Y} \eta t^{2}=\frac{F_{1} \cos \theta}{2} \\
& M=-\sigma_{Y} t \int_{-\frac{t}{2}}^{-\frac{\eta t}{2}} y d y+\sigma_{Y} t \int_{-\frac{\eta t}{2}}^{\frac{t}{2}} y d y=\frac{1}{4} \sigma_{Y} t^{3}\left(1-\eta^{2}\right)=\frac{F_{1} l \sin \theta}{4}
\end{aligned}
$$




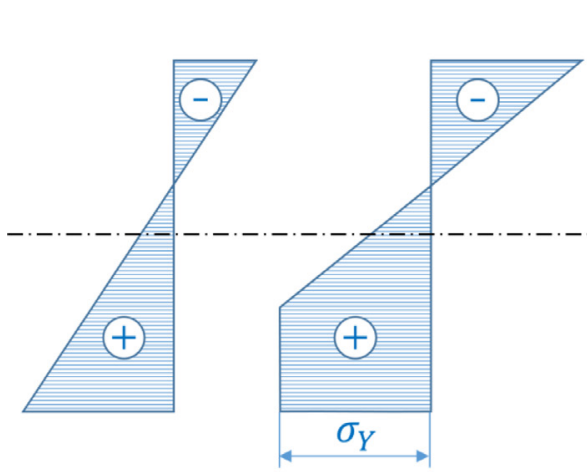

(a) (b)

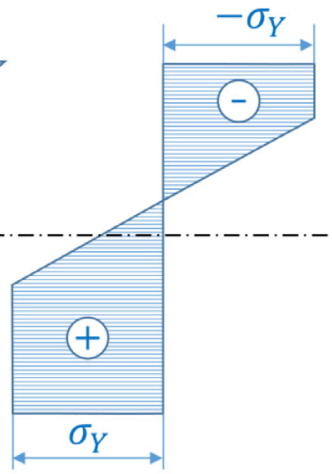

(c)

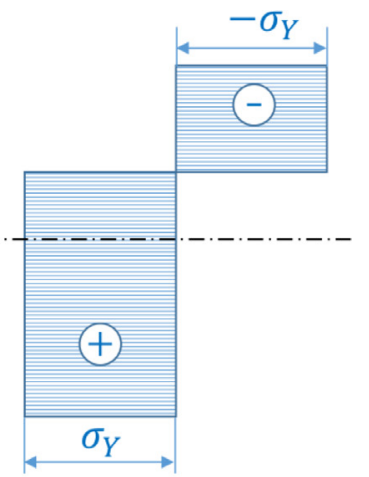

(d)

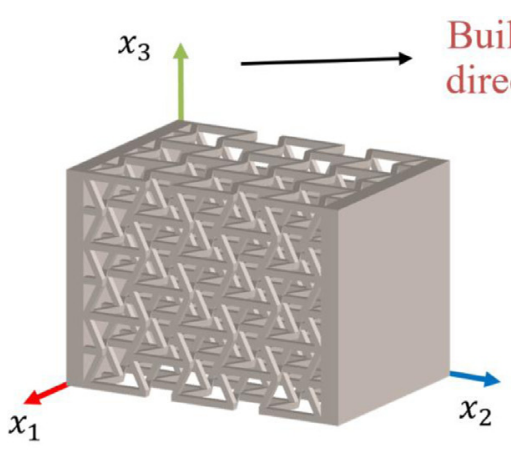

(a)

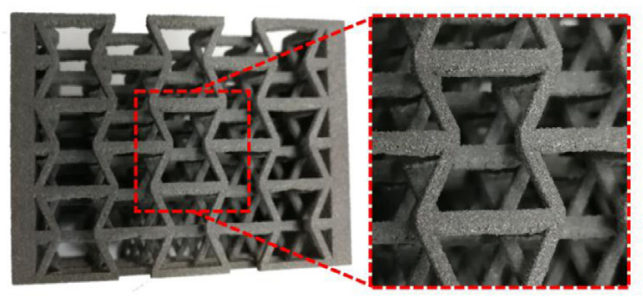

(c)

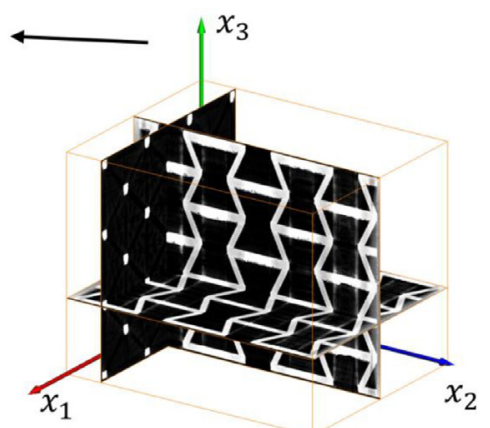

(b)

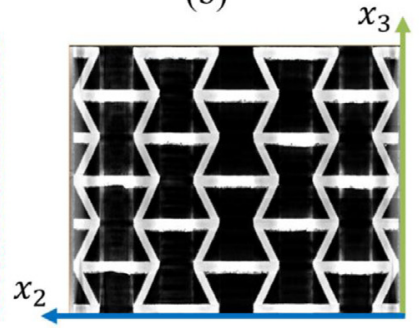

(d)

Fig. 3. Progression of yielding across the oblique strut's cross section.

Fig. 4. Building direction of the lattice structure; (a) CAD model, (b) tomography in three orthogonal plane, (c) Reentrant lattice sample printed by SLM, (d) tomography in $x_{2}-x_{3}$ plane.

Simultaneous Eqs. (4), (9) and (10) obtained:

$\sigma_{Y} t^{3}\left(1-\frac{\sigma_{o y}^{2} l^{4} \sin ^{4} \theta}{4 \sigma_{Y}^{2} t^{4}}\right)=\sigma_{o y} l^{3} \sin ^{3} \theta$

$\sigma_{o y}^{2} l^{4} \sin ^{4} \theta \cos ^{2} \theta+4 \sigma_{o y} \sigma_{Y} t l^{3} \sin ^{3} \theta-4 \sigma_{Y}^{2} t^{4}=0$

Accordingly, in the case of macroscopic yield of the oblique strut, ultimate strength $\sigma_{\text {oy }}$ applied far from the infinite 3D re-entrant lattice along z direction can be expressed as:

$\sigma_{o y}=\frac{2 \sigma_{Y} t\left(-l \sin \theta+\sqrt{l^{2} \sin ^{2} \theta+t^{2} \cos ^{2} \theta}\right)}{l^{2} \sin ^{2} \theta \cos ^{2} \theta}$

The final critical compressive strength of $3 \mathrm{D}$ re-entrant lattice is:

$\sigma_{c}=\min \left\{\sigma_{v b}, \sigma_{v y}, \sigma_{o y}\right\}$

\section{Experimental procedures and results discussion}

\subsection{Manufacturing and microstructure}

The unit cell of the 3D re-entrant structure investigated in this study is shown in Fig. 1. The shape of cross section of the struts in the auxetic cellular structure is square. The geometry parameters of the unit cell: the length of the vertical strut $h=11.875 \mathrm{~mm}$, the length of the re-entrant strut $l=6.987 \mathrm{~mm}$, the re-entrant angle between the vertical strut and the oblique strut $\theta=63.434^{\circ}$, and the thickness of the strut cross section $t=1.25 \mathrm{~mm}$. The samples contained three cell in $x_{1}, x_{2}$ and $x_{3}$ directions. The building direction and re-entrant sample printed by SLM are shown in Fig. 4.

For each experimental model, the specimens were manufactured via an Additive Manufacturing technology called Selective Laser Melting (SLM). The Al-Si10-Mg materials based in-situ micro-CT compression test samples used for this study were fabricated using an EOS M M290 (constructed by EOS GmbH, Germany) machine, a selective laser melting system from SLM Solutions GmbH. Raw material powder was obtained from EOS GmbH, certified with particle sizes ranging from 18 to $58 \mu \mathrm{m}$. AlSi10Mg fine powder was used for the production of these re-entrant samples. The SLM forming technology is using a high-energy laser beam to melt and fuse the metal powder together in a layer-bylayer manner to obtain an almost fully dense part. The SLM device is equipped with a laser power of $370 \mathrm{~W}$, a scanning speed of $1300 \mathrm{~mm} / \mathrm{s}$, a layer thickness of $30 \mu \mathrm{m}$ and an argon gas atmosphere. The designed samples are supported on a plate heated to $300{ }^{\circ} \mathrm{C}$. To relieve residual stresses generated by the fabrication process, thermal stress relieving was performed on these specimens at $300{ }^{\circ} \mathrm{C}$ for $2 \mathrm{~h}$, prior to removing specimens from the support structures.

Powder adhesion is a common defect in the formation of lightweight lattice structures. The presence of this defect affects the shape and 

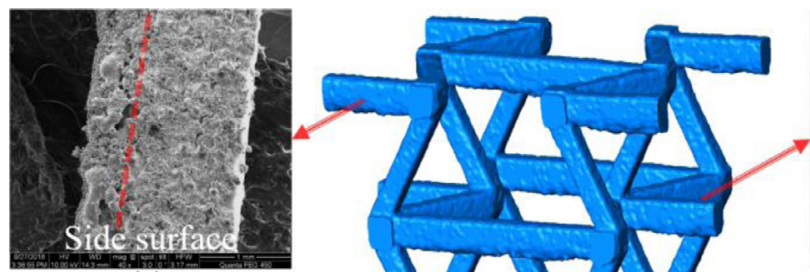

(c)

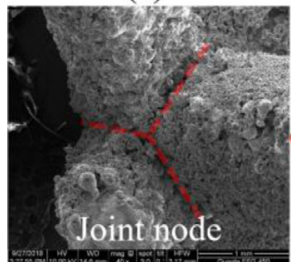

(e)

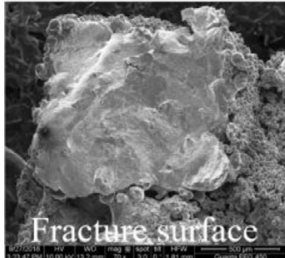

(g)

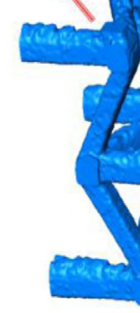

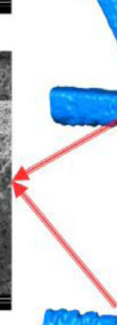

(a)

(a)

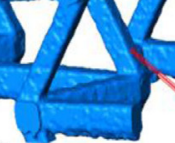

.

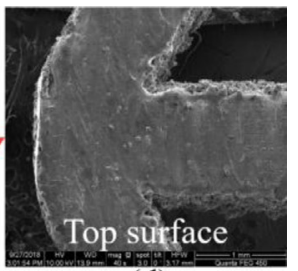

Fig. 5. Surface morphology; (a) The reconstructed unit cell. (b) Another perspective for observing the bottom surface. SEM images of (c) side surface, (d) top surface, (e) joint node, (f) oblique surface, $(\mathrm{g})$ fracture surface, $(\mathrm{h})$ bottom surface.

dimensional accuracy of the cores of lightweight lattice structure, and it is difficult to remove the adherent powder in the post-treatment stage. The generation of powder adhesion is related to the metal powder material and the forming structure. The high-energy laser beam spot selectively melts metal powder to form a melting pool and a heat-affected zone. The powder in heat-affected zone is insufficiently melted due to insufficient energy which result in the insufficiently melted metal powder adheres to the melting zone after cooling [41]. As shown in Fig. 5, we can see severe powder adhesion phenomenon on the surface of strut.

For horizontal or oblique struts, the roughness of top surface was barely affected by various powers and speeds of laser scanning (as shown in Fig. 5d), at the same time the roughness of bottom surfaces increased as the laser scanning power increased, and decreased as the laser scanning speed increased [12] (as shown in Fig. 5f and h).For horizontal struts, thermal flux will accumulate on the underside due to gravity, eventually causing excessive melting and lengthening the section of strut along the build direction [27]. The roughness of the top and bottom surfaces can be greatly different due to this local thermal behavior. The thermal flux is dissipated through the molten powder, which causes the bottom surface to be excessively melted and increase the roughness. The mechanism diagram of roughness formation is shown in Fig. 6.

\subsection{Mechanical properties of parent materials}

For parent materials AlSi10Mg, the mechanical parameters were measured by Digital Image Correlation (DIC) technique. The stressstrain curves of uniaxial tension specimens are shown in Fig. 7. The mechanical parameters can be directly obtained from these curves, as shown in Table 1: elastic modulus $E=33.2 \mathrm{GPa}$, yield stress at $0.2 \%$ plastic strain $\sigma_{Y}=260 \mathrm{MPa}$, ultimate strength $\sigma_{u}=466 \mathrm{MPa}$ and maximum plastic strain $\varepsilon_{p}^{\max }=4.2 \%$. It should be noted that although all uniaxial tension specimens were produced by the same printing process, their

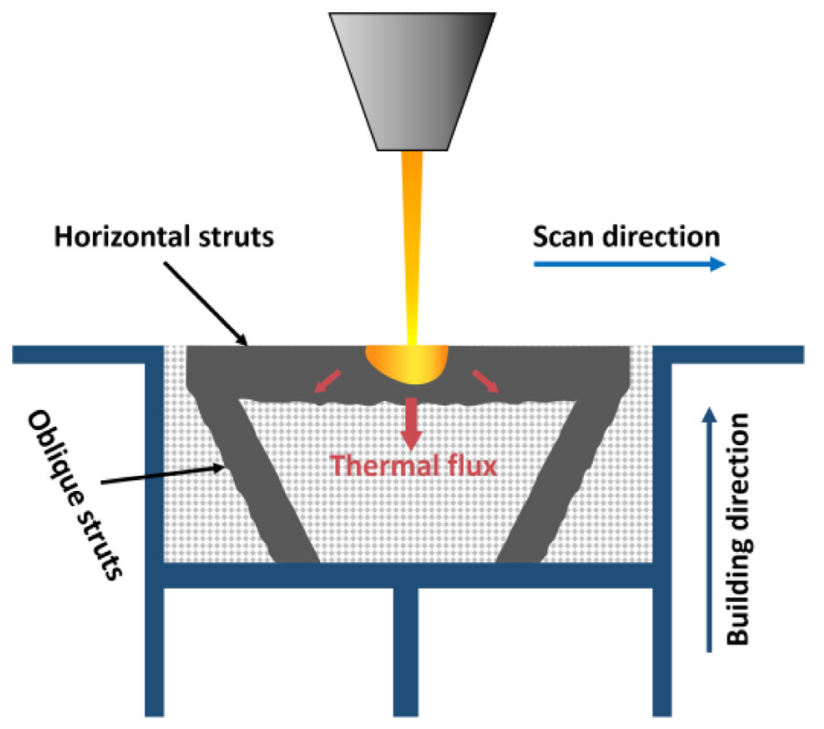

Fig. 6. Schematic view of roughness formation.

mechanical properties were different. The failure mode of the specimens was a brittle fracture, and no localized necking stage like that of aluminum alloy occurs. In fact, the influence of component size on mechanical parameters should be considered, but if the specimens have the same size with the struts in the as-built lattice structures, they will be too weak to test and be destroyed easily when clamping, it is difficult to measure their mechanical parameters. Therefore, block materials were used here. In the future research, different printing angles and different

Table 1

Loading steps.

\begin{tabular}{llll}
\hline Elastic modulus E & Yield stress $\sigma_{Y}$ & Ultimate strength $\sigma_{u}$ & Maximum plastic strain $\varepsilon_{p}^{\max }$ \\
\hline $33.2 \mathrm{GPa}$ & $260 \mathrm{MPa}$ & $466 \mathrm{MPa}$ & 1.0 \\
\hline
\end{tabular}




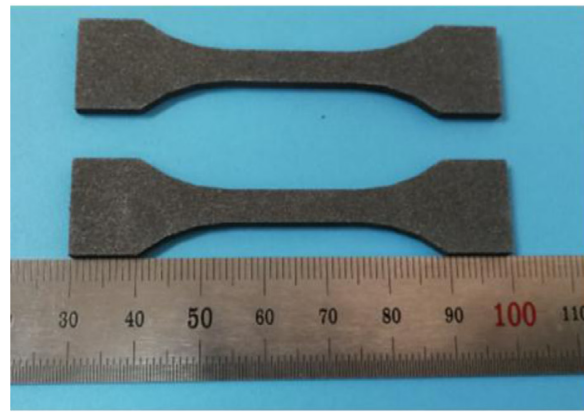

(a)

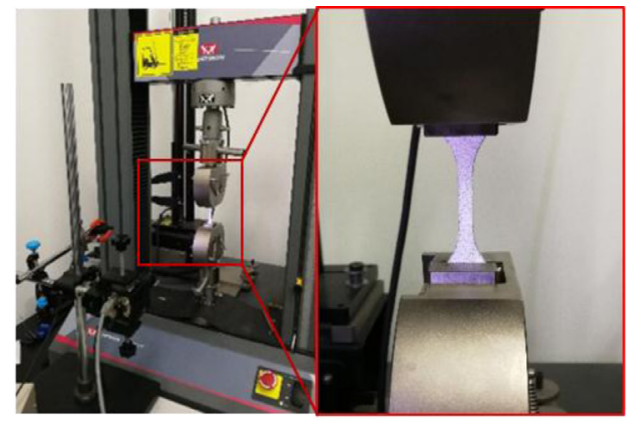

(b)

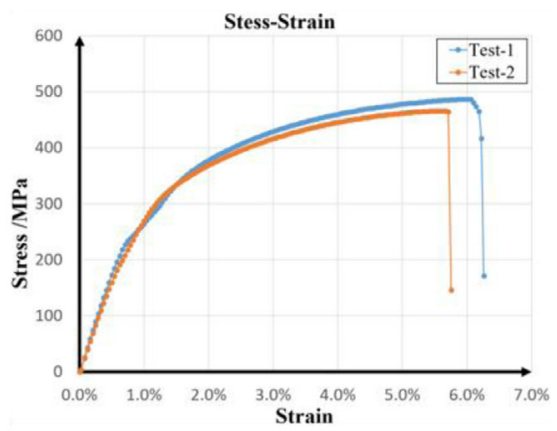

(c)

Fig. 7. (a) AlSi10Mg specimens; (b) DIC device and tensile test machine; (c)strain-stress curve.

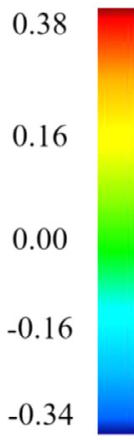

$\mathrm{mm}$

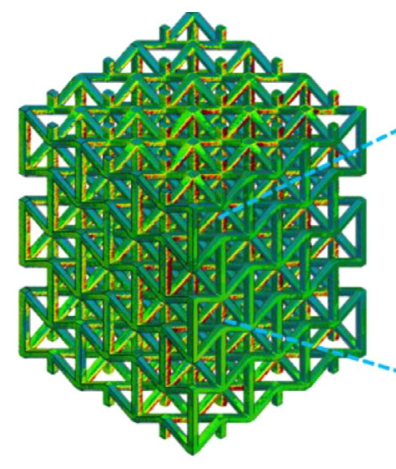

(a) Global tolerance

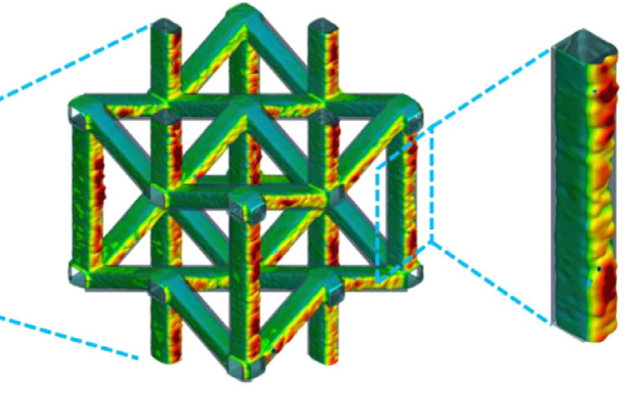

(b) Cell tolerance
Fig. 8. Comparison between 3D CT reconstruction model and CAD model.
Table 2

Loading steps.

\begin{tabular}{lllllllll}
\hline Step & 0 & 1 & 2 & 3 & 4 & 5 & 6 & 7 \\
\hline Displacement/mm & 0 & 0.5 & 1.0 & 1.5 & 2.0 & 2.5 & 3.3 & 4.5 \\
\hline
\end{tabular}

specimen size will be considered to study their influence on mechanical parameters of materials.

\subsection{In-situ CT experiment}

High-precision industrial X-ray computed tomography(CT)was used to perform 3D digital reconstruction of 3D additive manufacturing reentrant lattice to capture elastoplastic deformation, damage initiation and failure evolution of 3D lattice samples tested under in-situ compression experiments. The CT equipment operated at a voltage of $100 \mathrm{KV}$ and a tube current of $300 \mu \mathrm{A}$ was used to scan the lattice structure. During each scan, the sample was rotated from $0^{\circ}$ to $360^{\circ}$ at increments of $0.36^{\circ} / \mathrm{s}$ for each rotation step and a total set of 2048 images projected on the detector screen which has $2048 \times 2048$ square sensitive pixels was obtained after a full rotation.An isotropic voxel size of the reconstructed images was $29 \mu \mathrm{m}$. Zwick $Z 100$ device was used for the compression test, and the displacement rate of the pressure head was $0.5 \mathrm{~mm} / \mathrm{min}$ which is consistent with the ASTM standard E9-09.During each scan the displacement will stop for scanning and some relaxation was observed after each loading step. There were seven loading steps (as shown in Table 2) for observing the mechanical behavior of the re-entrant lattice structure.

Avizo ${ }^{\circledR}$ software was used to perform the Image noise reduction, image segmentation, three dimensional visualization and quantification. First of all, apply the median filter to the tomography data to remove noise for improving the results of later processing (for example, edge detection on images). Then each 3D tomographic dataset was segmented into two white color represented as the solid material and black color represented as large voids of the lattice structure by a single threshold value, and morphological operation was implemented to remove small isolated points that have no relationship with the material points. Finally, 3D surface mesh model was generated from 3D binary dataset.

\subsubsection{Geometric error}

After the 3D surface mesh model (CT model) was generated, it was necessary to perform a three dimensional comparison between the CT model and CAD model for getting the geometric errors of the 3Dreentrant lattice structure. For the purpose of doing this comparison, the two models could be aligned through fast and automatic best fit alignment tool or manual alignment tool. In Fig. 8, all the dimensional differences between the CAD model created by SolidWorks ${ }^{\circledR}$ and the actual CT reconstructed surface (STL mesh) are represented by colors. The global tolerance ranges from $-0.34 \mathrm{~mm}$ to $0.38 \mathrm{~mm}$ and the thickness of a single ideal CAD strut is $1.25 \mathrm{~mm}$, that means for a single strut the maximum geometrical tolerance is $-27.2 \%$ to $30.4 \%$ which has significant influence on the local deformation or failure mode.

\subsubsection{Deformation response}

In this study, the real-time three-dimensional deformation behavior of 3D re-entrant lattice structure was studied by simultaneously tracking microscopic and macroscopic scale deformations. The curve of force versus displacement is shown in Fig. 9(a). The numbers correspond to each loading step were marked on each plot. As mentioned above, the information including 3D spatial location of each joint node and strut about the as-built lattice was provided by each 3D tomographic dataset. Local deformation was quantified and failure mechanisms were identified through time tracking. Fig. 9 (c) shows the overall failure process of the re-entrant lattice which was reconstructed from the tomography at each loading step. Red circles manifest that the edge struts (at lattice boundary) failure from buckling to fracture. 


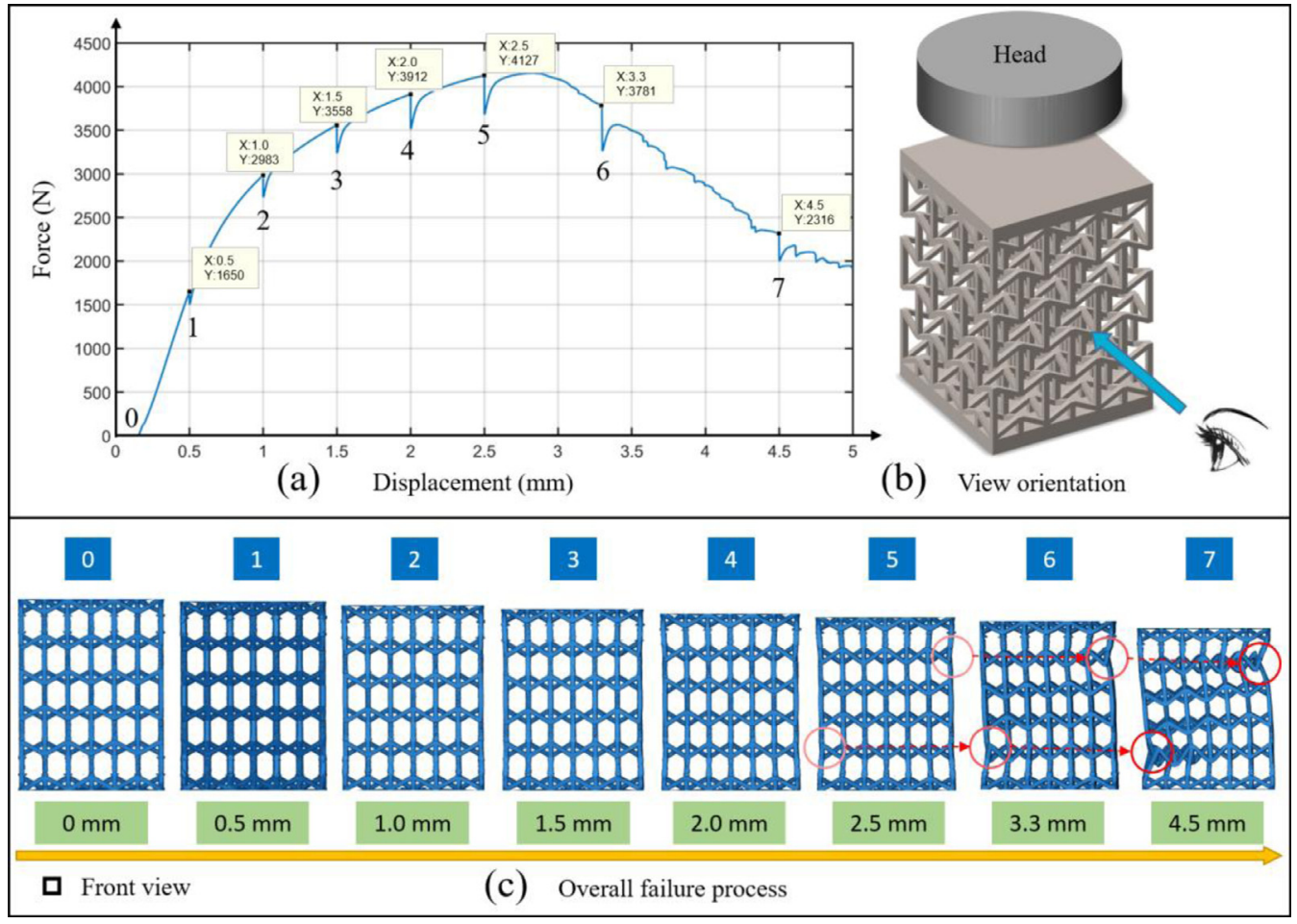

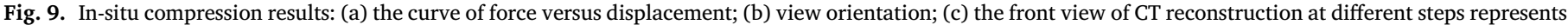
overall failure process of re-entrant lattice.

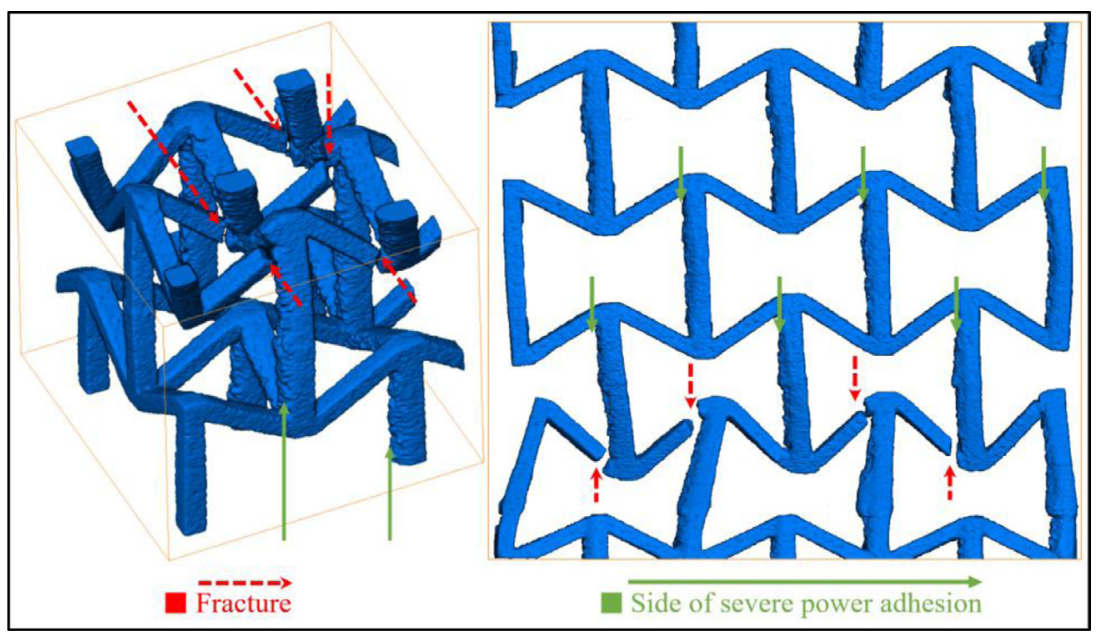

Fig. 10. Sub-volumes of the 7th reconstruction model, red dashed arrows indicate the location of fracture and green solid arrows indicate the side of severe powder adhesion.

\subsubsection{Tomography analysis}

In order to observe the fracture position of struts clearly, the subvolumes were extracted from the 3D tomographic dataset of 7th loading step, as shown in Fig. 10. The most obvious finding to emerge from Fig. 10 is that the occurrence of fracture location of strut tends to the side of severe powder adhesion. The reason for this phenomenon is that excessive melting and powder adhesion have an effect on the strength of the joint node which is a significant contributory factor to the fracture location of strut.

As mentioned previously, the oblique strut in the lattice is subjected to tension, bending, and shearing, but it has been found through experiments that the deformation of the strut is also accompanied by torsion. This is mainly due to the lower nodal connectivity of the struts at external boundaries than those at the interior. Additional boundary effects exacerbate nodal rotations, leading to the introduction of torsion in the deformation of struts intersecting at lattice corners. Fig. 11 shows the deformation process of the struts in the boundary cell over time during loading.

Then, sub-volumes around the joint nodes were extracted to inspect the failure history of node. Owing to the nodes in the 0-3 steps have no obvious deformation, only the regions around the nodes in 4-7 steps were intercepted to display. As can be seen from Fig. 12, the rupture initiation of the struts was located outside the re-entrant angle.

In the boundary cell, the fracture of oblique struts under the combined action of the stretching, bending, shearing, and torsion occurred ahead of the buckling of vertical struts. Meanwhile, the vertical strut was eccentrically bent due to the twist of the node. In the interior cell of the lattice structure, vertical struts did not undergo buckling, and the yield of oblique struts started firstly. According to Eqs. (6), (8) and (10): $\sigma_{v b}=2.926 \mathrm{MPa}, \sigma_{v y}=5.200 \mathrm{MPa}, \sigma_{o y}=2.075 \mathrm{MPa}, \sigma_{o y}<\sigma_{v b}<\sigma_{v y}$, which is consistent with the experimental results. 
- Step0

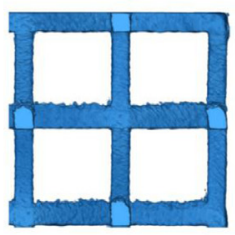

- Step1
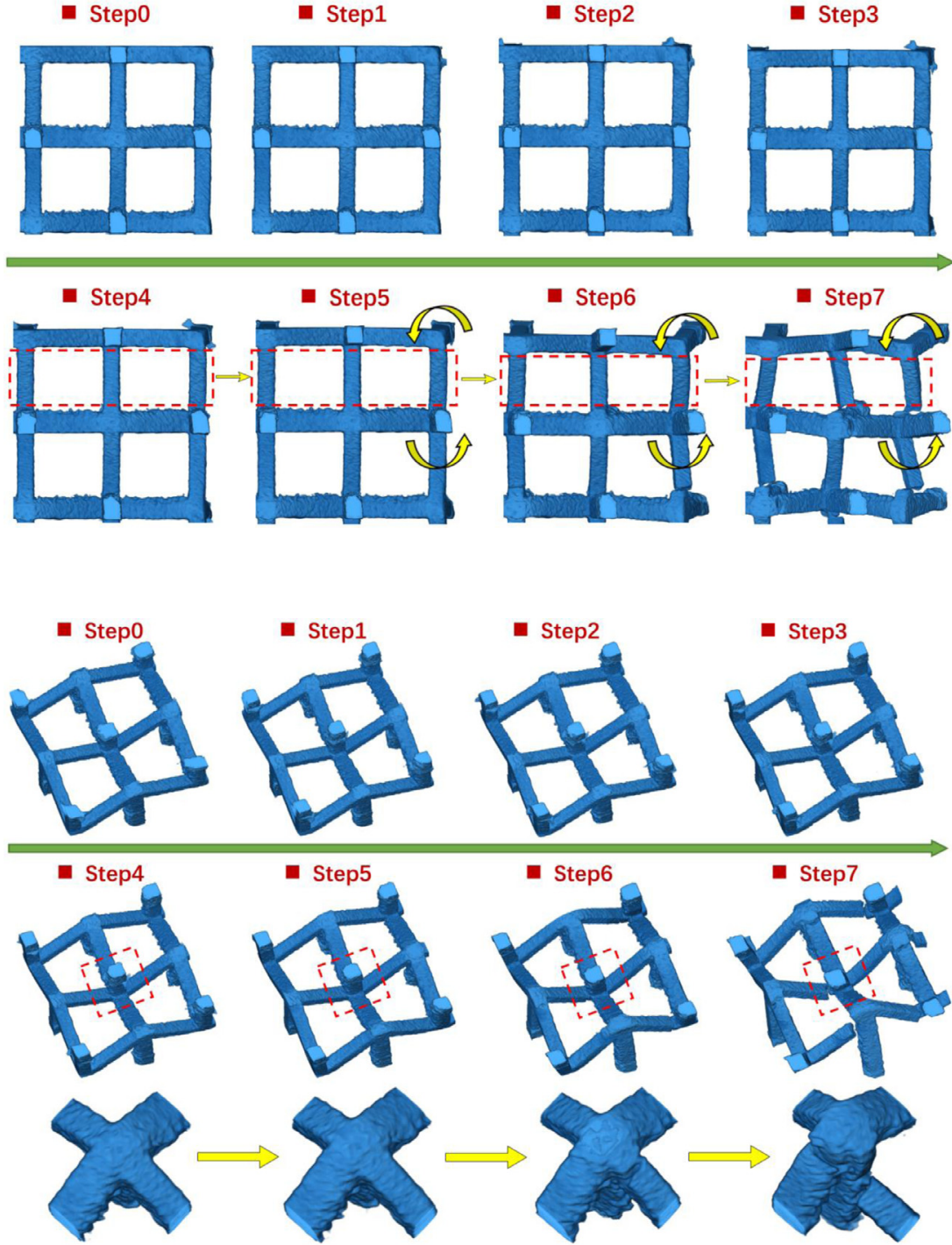

Fig. 11. Subvolumes of each 3D reconstruction model, red dashed rectangles manifest the evolution of the deformation of the struts, the yellow arrows indicate the rotation of the node.

Fig. 12. Sub-volumes of nodes in 4-7 steps.

\section{Numerical simulation and results discussion}

\subsection{FEA of single struts}

After analyzing the experimental test, Finite Element Analysis (FEA) was performed to compare the result. First of all, for the sake of easy analysis, the results of FEA of the single strut with ideal geometry (named CAD model) and the single strut with real geometry (named CT reconstructed model) were compared, as shown in Fig. 13.

The ideal strut of $9 \mathrm{~mm}$ long was meshed into 28,928 eight-node linear hexahedron elements using ABAQUS ${ }^{\circledR}$. The CT reconstructed strut of $9 \mathrm{~mm}$ long was meshed into 134,956 ten-node quadratic tetrahedron elements. The same boundary conditions were imposed on the mesh of the struts: all displacement degree of freedom of all nodes located on the left surface was constrained blocked, axial displacement degree of freedom of all nodes located on the right surface was subjected to an imposed displacement of $5 \mathrm{~mm}$. The classical metal plasticity models were implemented in the material model using Mises yield surface with J2 plastic flow, which allows for isotropic hardening. And, the ductile damage criterion was applied to the material model.

For the FEA of CAD model, the strut was regular with lower strain localizations and more homogeneous damage than the CT reconstructed model. Load-displacement curves and stress-strain curves for the dif- ferent FE model of single struts were shown in Fig. 14. Because the cross section caused by excessive melting increased, the ultimate load of the CT reconstruction model was higher than the CAD model, but the ultimate stress of the CT reconstruction model was lower than the CAD model. Since the cross-section of the model reconstructed by CT was variable, the average of 20 cross-sections was taken to calculate its stress-strain curve.

\subsection{FEA of $3 D$ re-entrant lattices}

Moving on now to consider the whole re-entrant structure. The first FE model that is based on an ideal geometry of the specimen was meshed into 101,709 eight-node linear hexahedron elements. The second FE model of the real structure defined from the tomography, was then meshed into 2, 390,457 four-node tetrahedral elements. Although the same type of element should be selected for numerically analysis for fairness, we used different element types for CAD model, only the LoadDisplacement curves have a little difference, the results are almost the same. For the higher accuracy and lower computational burden of hexahedron element, we chose and showed the result of the hexahedron element of CAD model.

A uniaxial compression loading was applied on the generated volume meshes. The typical boundary conditions imposed on the mesh of the CT 


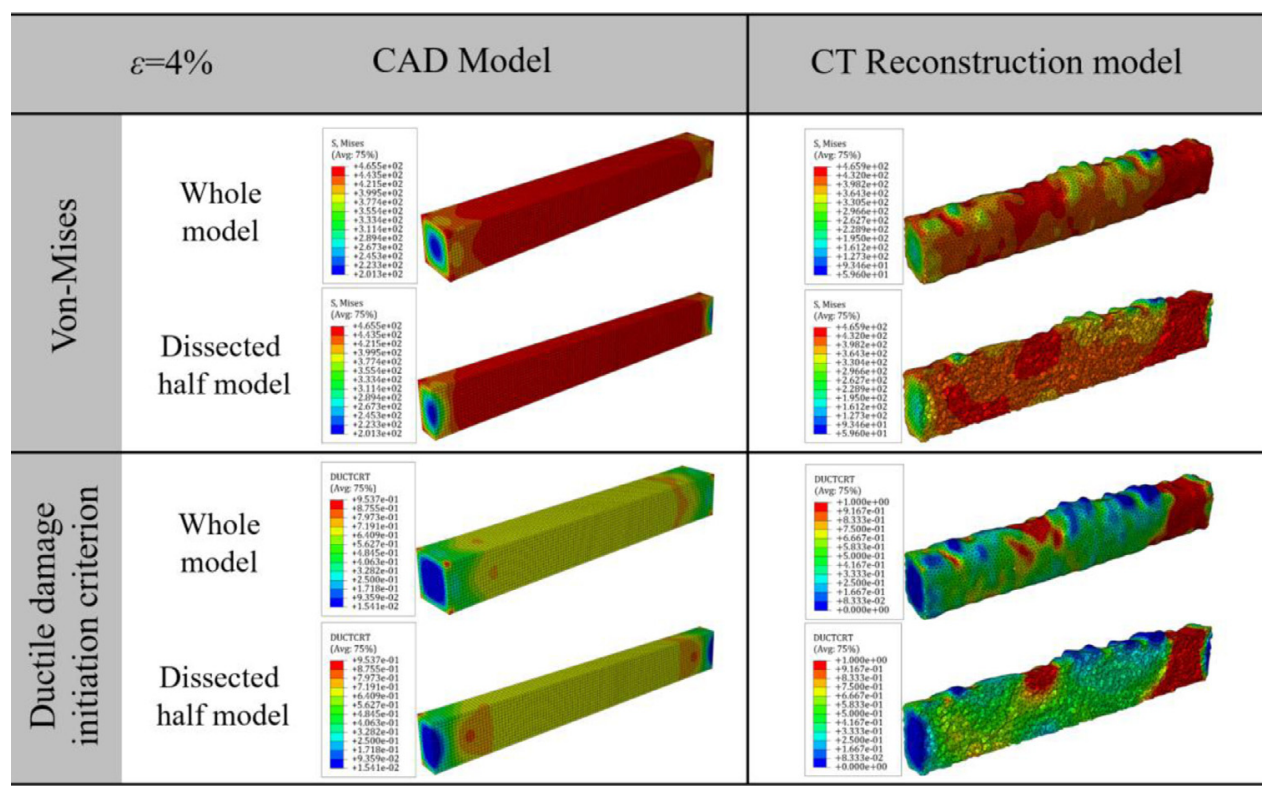

Fig. 13. Numerical simulation results presented for single struts: FEA of CAD model (left), FEA of CT reconstructed model(right).

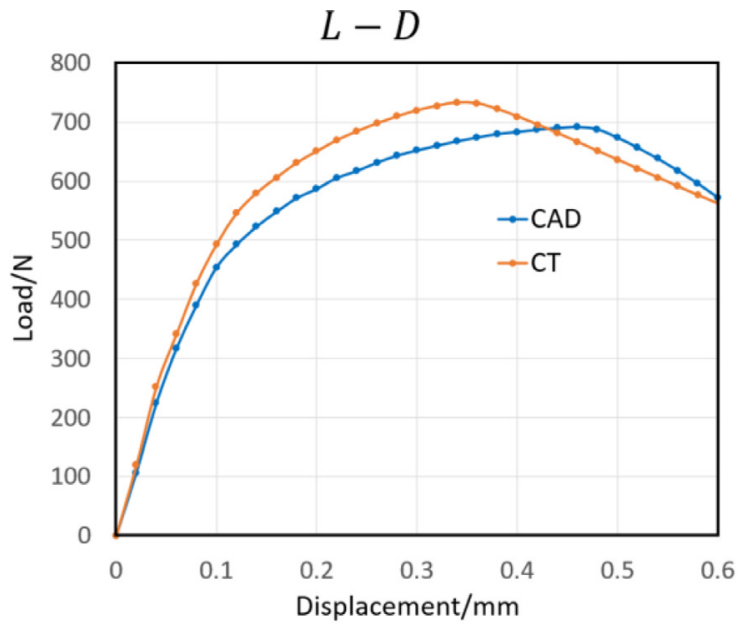

(a)

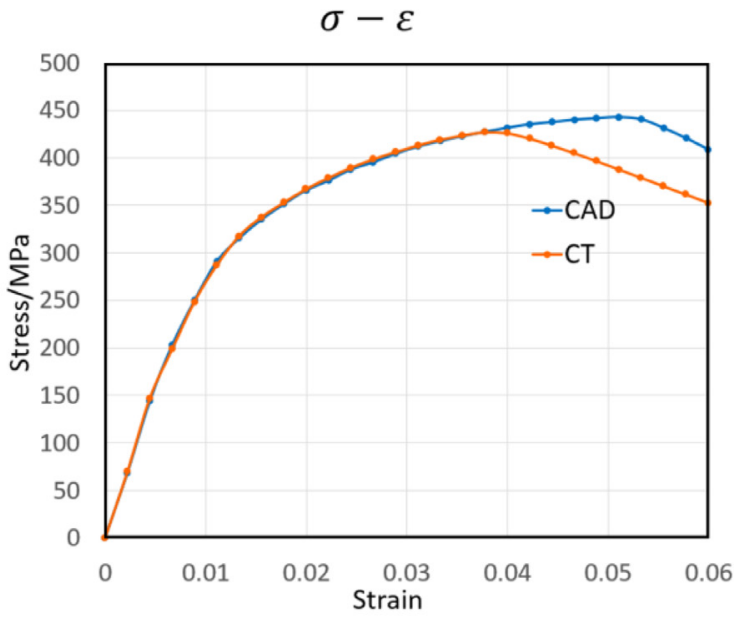

(b)

Fig. 14. FEA results of single CT reconstructed strut and CAD strut: (a) Load-displacement curves; (b) stress-strain curves.

reconstruction model were shown Fig. 15. The bottom head was blocked and the top head was subjected to an imposed displacement of $5 \mathrm{~mm}$. All degrees of freedom of the point 1 was blocked, point 2 was blocked in the $x$ direction and point 3 was blocked in the $y$ direction to prevent global rotation about the compression axis. Because explicit dynamic procedure caused too much time, implicit dynamic procedure was created to analysis this problem. Identical boundary conditions were imposed on the model of the ideal CAD model but explicit dynamic procedure was adopted to perform FEA.

The FEA results of the CT reconstructed model and the CAD model at linear elastic stage were compared, as shown in Fig. 16. And the predicted load-displacement curves of the ideal CAD and CT reconstructed model were compared to the experimental curve in Fig. 17. In contrast with experimental result, it is clear that the FE simulations of the ideal CAD and CT reconstructed model result in overestimated load values. Fig. 18 shows the final failure mode of the CAD model, which is consistent with the experimental results. But the failure results of the CT reconstruction model have not been shown here due to computational efficiency issues. Owing to some limitations of the FE model, numerical results definitely will have some difference with experimental result.
In fact, it is difficult to run a convergence analysis on account of the computational burden of the geometrical reconstruction. And enormous numbers of mesh and the scarcity of computer memory refused to adopt a mesh with quadratic elements.

The numerical analysis results indicate that: (1)The stress concentration area at the intersection of struts with real geometry is significantly larger than the ideal CAD model and strain localizations is more obvious; (2) Geometric irregularities can lead to a reduction in critical buckling load and the surface morphology of specimen reduces the ultimate strength and strain at break of the solid material; (3) The reason why FE simulations with the ideal CAD model results in higher load values compared to experiments is that the ideal model have perfect geometry; (4) FE simulations with the CT reconstructed model results in higher load values compared to CAD model and experiments, that is because the powder adhesion caused by over-melting hardly participates in load-bearing, but this part of can't be distinguished by CT technology, which leads to the existence of fictitious bearing area in the finite element model, and finally the load calculated by the reconstructed model is artificially high; (5) Surface roughness and powder adhesion do not affect the final failure mode of 3D re-entrant lattice structure. 
Top head

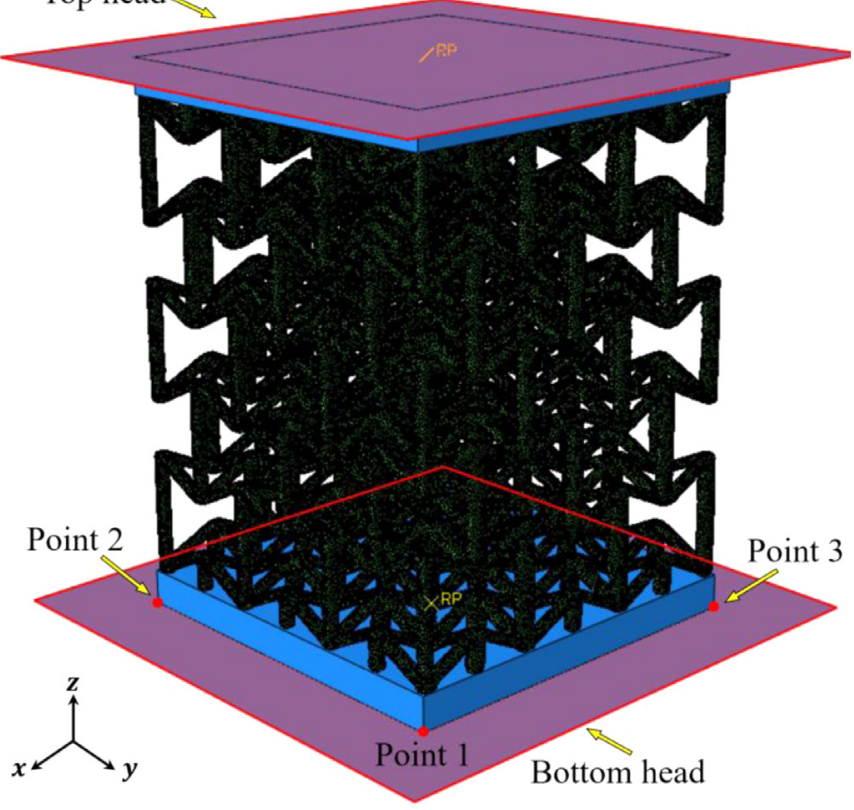

Fig. 15. Boundary conditions imposed on the CT reconstruction model.

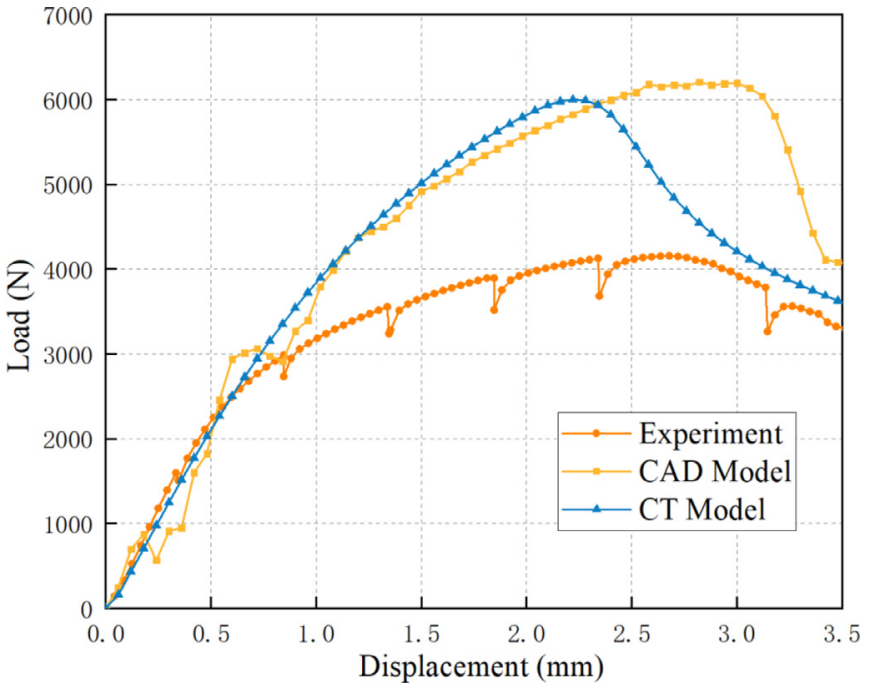

Fig. 17. Load-displacement curves obtained by FE simulations of the CAD model and CT reconstructed model and compression test.

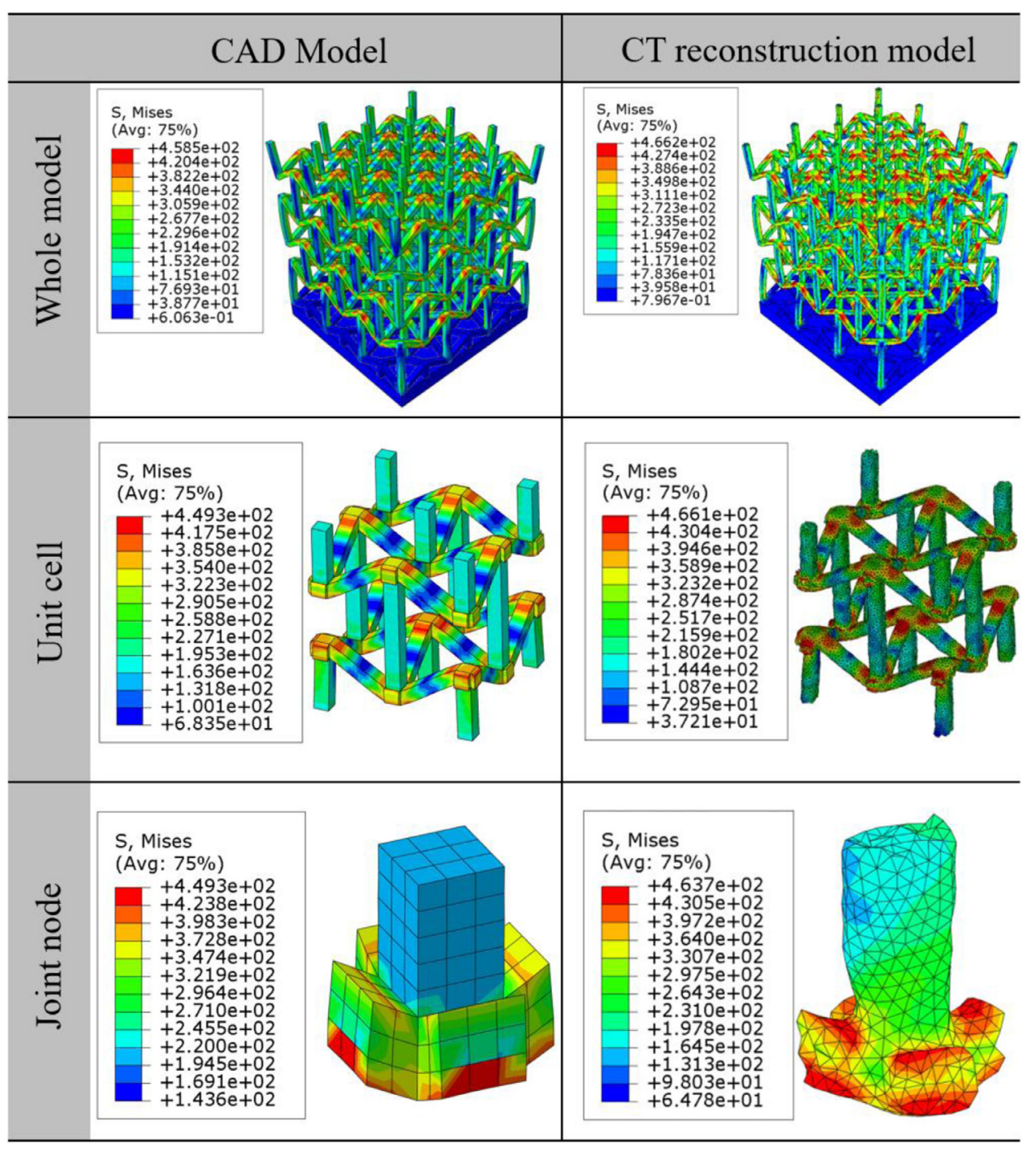

Fig. 16. Numerical simulation results presented for the whole structure: FEA of CAD model (left), FEA of CT reconstructed model(right). 


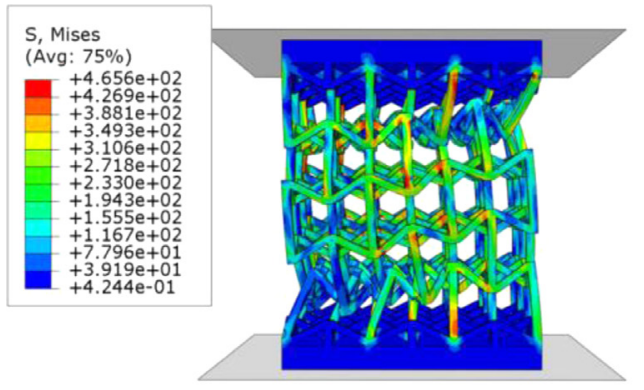

(a)

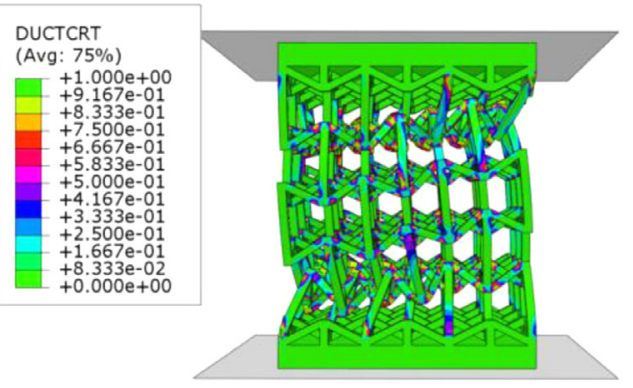

(b)

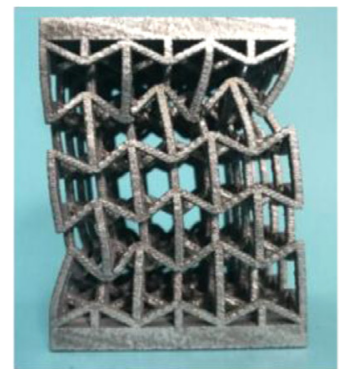

(c)

Fig. 18. FEA results the CAD model: (a) Von Mises stress field of final step, (b) damage initiation field of final step. (c) experimental result.

\section{Conclusion}

In this work, theoretical models for predicting the mechanical properties of 3D re-entrant lattice are developed, such as stiffness, Poisson's ratio and strength, etc. Afterwards, 3D re-entrant lattices with negative Poisson's ratio were manufactured by SLM, and in-situ X-ray computed tomography (CT) is used to obtain the shape, position and distribution of defects to study their mechanical response, damage initiation and failure evolution under quasi-static compression. The geometric defects extracted from the tomographic images are introduced into the finite element model and the corresponding numerical simulation of the compression test is carried out to predict the deformation behavior of lattice structures. From the aspects of elastic modulus, strength and failure mechanism, it reveals the influence of defects on the 3D reentrant lattice structures. The insights gained from the experimental results and FEA results demonstrate the following conclusions:

1 Geometric errors between the samples and the CAD models are large, which have a significant impact on the mechanical properties of the structures and joint nodes resulting in the fracture failure of the struts tends to occur on the side where the powder adhesion is serious.

2 In the interior cell of the lattice structure, vertical struts did not undergo buckling and the yield of oblique struts started firstly based on the analytical solution of critical compressive strength.

3 The 3D re-entrant lattice with real geometry shows higher stress concentration and more obvious strain localizations. Geometric irregularities of samples reduce the ultimate strength and strain at break of the solid material.

4 Both FEA results of CAD model and CT reconstructed model exhibit higher load values compared to experiments. The CT model with real geometry has fictitious bearing area which hardly participates in load-bearing.

In addition, the FE analysis on the real structure does not remove the part which hardly participates in load-bearing. The fictitious bearing area might be required to be considered since which results in increasing bearing area and predictive load values. The modelling of the fictitious bearing area will be considered for future developments.

\section{Acknowledgements}

This research is supported by the Foundation for Innovative Research Groups of the National Natural Science Foundation of China (Grant no. 11521202), the Project of State Key Laboratory of Explosion Science and Technology.

\section{References}

[1] Scarpa F, Tomlin PJ. On the transverse shear modulus of negative Poisson's ratio honeycomb structures. Fatigue Fract Eng M 2000;23(8):717-20.

[2] Scarpa F, Ciffo LG, Yates JR. Dynamic properties of high structural integrity auxetic open cell foam. Smart Mater Struct 2004;13(1):49-56 2004-01-01.
[3] Prawoto Y. Seeing auxetic materials from the mechanics point of view: a structural review on the negative Poisson's ratio. Comput Mater Sci 2012;58:140-53.

[4] Lira C, Scarpa F, Rajasekaran R. A gradient cellular core for aeroengine fan blades based on auxetic configurations. J Intel Mater Syst STR 2011;22(9):907-17.

[5] Kuribayashi K, Tsuchiya K, You Z, Tomus D, Umemoto M, Ito T, et al. Self-deployable origami stent grafts as a biomedical application of Ni-rich TiNi shape memory alloy foil. Mater Sci Eng: A 2006;419(1-2):131-7.

[6] Ali MN, Rehman IU. An Auxetic structure configured as oesophageal stent with potential to be used for palliative treatment of oesophageal cancer; development and in vitro mechanical analysis. J Mater Sci 2011;22(11):2573-81.

[7] Ko J, Bhullar S, Cho Y, Lee PC, Byung-Guk Jun M. Design and fabrication of auxetic stretchable force sensor for hand rehabilitation. Smart Mater Struct 2015;24(7):75027 2015-01-01.

[8] Kempen K, Thijs L, Van Humbeeck J, Kruth JP. Mechanical properties of AlSi10Mg produced by selective laser melting. Phys Procedia 2012;39:439-46.

[9] Rosenthal I, Stern A, Frage N. Microstructure and mechanical properties of AlSi10Mg parts produced by the laser beam additive manufacturing (AM) technology. Metallogr Microstruct Anal 2014;3(6):448-53.

[10] Galy C, Le Guen E, Lacoste E, Arvieu C. Main defects observed in aluminum alloy parts produced by SLM: from causes to consequences. Addit Manuf 2018;22:165-75.

[11] Leon A, Aghion E. Effect of surface roughness on corrosion fatigue performance of AlSi10Mg alloy produced by Selective Laser Melting (SLM). Mater Charact 2017;131:188-94.

[12] Tian Y, Tomus D, Rometsch P, Wu X. Influences of processing parameters on surface roughness of Hastelloy X produced by selective laser melting. Addit Manuf 2017;13:103-12.

[13] Lecarme L, Maire E, Kumar KCA, De Vleeschouwer C, Jacques L, Simar A, et al. Heterogenous void growth revealed by in situ 3-D X-ray microtomography using automatic cavity tracking. Acta Mater 2014;63:130-9.

[14] Maire E, Bouaziz O, Di Michiel M, Verdu C. Initiation and growth of damage in a dual-phase steel observed by X-ray microtomography. Acta Mater 2008;56(18):4954-64.

[15] Takano N, Takizawa H, Wen P, Odaka K, Matsunaga S, Abe S. Stochastic prediction of apparent compressive stiffness of selective laser sintered lattice structure with geometrical imperfection and uncertainty in material property. Int J Mech Sci 2017;134:347-56.

[16] Leary M, Mazur M, Williams H, Yang E, Alghamdi A, Lozanovski B, et al. Inconel 625 lattice structures manufactured by selective laser melting (SLM): mechanical properties, deformation and failure modes. Mater Des 2018;157:179-99.

[17] Bernal Ostos J, Rinaldi RG, M Hammetter C, Stucky GD, Zok FW, Jacobsen AJ. Deformation stabilization of lattice structures via foam addition. Acta Mater 2012;60(19):6476-85

[18] Hernández-Nava E, Smith CJ, Derguti F, Tammas-Williams S, Leonard F, Withers PJ, et al. The effect of defects on the mechanical response of Ti-6Al-4V cubic lattice structures fabricated by electron beam melting. Acta Mater 2016;108:279-92.

[19] Boniotti L, Beretta S, Foletti S, Patriarca L. Strain concentrations in BCC micro lattices obtained by AM. Procedia Struct Integr 2017;7:166-73.

[20] Kerckhofs G, Pyka G, Moesen M, Van Bael S, Schrooten J, Wevers M. High-resolution microfocus X-Ray computed tomography for 3D surface roughness measurements of additive manufactured porous materials. Adv Eng Mater 2013;15(3):153-8.

[21] Melancon D, Bagheri ZS, Johnston RB, Liu L, Tanzer M, Pasini D. Mechanical characterization of structurally porous biomaterials built via additive manufacturing: experiments, predictive models, and design maps for load-bearing bone replacement implants. Acta Biomater 2017;63:350-68.

[22] Crupi V, Kara E, Epasto G, Guglielmino E, Aykul H. Static behavior of lattice structures produced via direct metal laser sintering technology. Mater Des 2017;135:246-56

[23] Carlton HD, Lind J, Messner MC, Volkoff-Shoemaker NA, Barnard HS, Barton NR, et al. Mapping local deformation behavior in single cell metal lattice structures. Acta Mater 2017;129:239-50.

[24] Liu L, Kamm P, García-Moreno F, Banhart J, Pasini D. Elastic and failure response of imperfect three-dimensional metallic lattices: the role of geometric defects induced by Selective Laser Melting. J Mech Phys Solids 2017;107:160-84.

[25] Amani Y, Dancette S, Delroisse P, Simar A, Maire E. Compression behavior of lattice structures produced by selective laser melting: X-ray tomography based experimental and finite element approaches. Acta Mater 2018;159:395-407. 
[26] Bagheri ZS, Melancon D, Liu L, Johnston RB, Pasini D. Compensation strategy to reduce geometry and mechanics mismatches in porous biomaterials built with Selective Laser Melting. J Mech Behav Biomed 2017;70:17-27.

[27] Suard M, Martin G, Lhuissier P, Dendievel R, Vignat F, Blandin JJ, et al. Mechanical equivalent diameter of single struts for the stiffness prediction of lattice structures produced by Electron Beam Melting. Addit Manuf 2015;8:124-31.

[28] Wang B, Zhu S, Hao P, Bi X, Du K, Chen B, et al. Buckling of quasi-perfect cylindrical shell under axial compression: a combined experimental and numerical investigation. Int J Solids Struct 2018;130-131:232-47.

[29] Hao P, Wang B, Tian K, Du K, Zhang X. Influence of imperfection distributions for cylindrical stiffened shells with weld lands. Thin Wall Struct 2015;93:177-87.

[30] Schwerdtfeger J, Wein F, Leugering G, Singer RF, Körner C, Stingl M, et al. Design of auxetic structures via mathematical optimization. Adv Mater 2011;23(22-23):2650-4 2011-06-17.

[31] Yang L, Harrysson O, West H, Cormier D. Compressive properties of Ti-6Al-4V auxetic mesh structures made by electron beam melting. Acta Mater 2012;60(8):3370-9.

[32] Wang K, Chang Y, Chen Y, Zhang C, Wang B. Designable dual-material auxetic metamaterials using three-dimensional printing. Mater Des 2015;67:159-64.

[33] Wang X, Li X, Ma L. Interlocking assembled 3D auxetic cellular structures. Mater Des 2016;99:467-76.
[34] Xue Y, Wang X, Wang W, Zhong X, Han F. Compressive property of Al-based auxetic lattice structures fabricated by 3-D printing combined with investment casting. Mater Sci Eng: A 2018;722:255-62.

[35] Wang X, Chen Y, Ma L. The manufacture and characterization of composite three-dimensional re-entrant auxetic cellular structures made from carbon fiber reinforced polymer. J Compos Mater 2018;52(23):3265-73.

[36] Yang L, Harrysson O, West H, Cormier D. Mechanical properties of 3D re-entrant honeycomb auxetic structures realized via additive manufacturing. Int J Solids Struct 2015;69-70:475-90.

[37] Yang L, Harrysson O, West H, Cormier D. Modeling of uniaxial compression in a 3D periodic re-entrant lattice structure. J Mater Sci 2013;48(4):1413-22.

[38] Wang X, Wang B, Li X, Ma L. Mechanical properties of 3D re-entrant auxetic cellular structures. Int J Mech Sci 2017;131-132:396-407.

[39] Li X, Lu Z, Yang Z, Yang C. Directions dependence of the elastic properties of a 3D augmented re-entrant cellular structure. Mater Des 2017;134:151-62.

[40] Gibson LJ, Ashby MF. Cellular solids: structure and properties. Cambridge university press; 1999.

[41] Tan JH, Wong WLE, Dalgarno KW. An overview of powder granulometry on feedstock and part performance in the selective laser melting process. Addit Manuf 2017;18:228-55. 